\title{
FUNÇÃO GERADORA E EQUAÇÃO CARACTERÍSTICA NO CONTEXTO DE INVESTIGAÇÃO HISTÓRICA DO MODELO COMPLEXO DE FIBONACCI FUNDAMENTADA NA ENGENHARIA DIDÁTICA
}

\section{GENERATING FUNCTION AND CHARACTERISTIC EQUATION IN THE CONTEXT OF HISTORICAL RESEARCH OF THE COMPLEX MODEL OF FIBONACCI FOUNDED IN DIDACTIC ENGINEERING}

\author{
Rannyelly Rodrigues de Oliveira ${ }^{1}$ \\ Instituto Federal de Educação, Ciência e Tecnologia do Ceará - IFCE \\ Maria Helena de Andrade ${ }^{2}$ \\ Instituto Federal de Educação, Ciência e Tecnologia do Ceará - IFCE \\ Francisco Régis Vieira Alves ${ }^{3}$ \\ Instituto Federal de Educação, Ciência e Tecnologia do Ceará - IFCE
}

\section{Resumo}

Este trabalho apresenta um experimento didático realizado na disciplina de História da Matemática (HM) no curso de Licenciatura em Matemática do Instituto Federal de Educação, Ciência e Tecnologia do Ceará (IFCE). Este experimento é parte da pesquisa que está em andamento no Mestrado Acadêmico do programa de Pós-Graduação em Ensino de Ciências e Matemática (PGECM/IFCE). A presente pesquisa aborda uma temática, sobre representações complexas do modelo de Fibonacci (MF), que busca oportunizar uma perspectiva epistemológica no contexto da HM e na formação docente. Por outro lado, compreende-se a existência de uma problemática, em que os livros de HM apresentam uma abordagem sucinta, lúdica e escassa das relações matemáticas Fibonaccianas, pouco relevante para que o leitor compreenda o processo evolutivo do MF a partir da descrição do modelo biológico da reprodução de coelhos. Numa perspectiva epistemológica, essa pesquisa tem como objeto de investigação a função geradora e a equação característica, ambas são discutidas na classe das estruturas polinomiais do MF que compõem um repertório inerente a sua complexificação a partir da inserção de variáveis e da unidade imaginária "i”. Numa abordagem didática e cognitiva, tem-se o propósito de trabalhar essas relações matemáticas em situações de ensino. Para isso, segue-se o percurso metodológico fundamentado na Engenharia Didática (ED) que é intrínseca das pesquisas no ensino de Matemática e que é realizada em situações didáticas amparada na aplicação de situações-problema. Além do mais, a ED tem como pressupostos elementos de ordem epistemológica, didática e cognitiva, assim, permitindo explorar propriedades matemáticas nas aulas de HM. Por fim, constatou-se uma validação interna da pesquisa referente à compreensão da função geradora e equação característica. Além do mais, foi possível observar que alguns

\footnotetext{
${ }^{1}$ nanny-rockstar@hotmail.com

2 helenaeducadoramat@gmail.com

3 fregis@ifce.edu.br
} 
alunos, docentes em formação inicial, desenvolveram uma compreensão da evolução histórica e matemática da Sequência de Fibonacci.

Palavras-chave: Engenharia Didática; História da Matemática; Formação docente; Modelo complexo de Fibonacci; Função geradora.

\begin{abstract}
This work presents a didactic experiment carried out in the Mathematics History (HM) course in the Mathematics Degree course of the Instituto Federal de Educação, Ciência e Tecnologia do Ceará (IFCE). This experiment is part of the research that is underway in the Academic Master of the Graduate Program in Teaching Science and Mathematics (PGECM / IFCE). The present research deals with a thematic about complex representations of the Fibonacci (MF) model, which seeks to provide an epistemological perspective in the context of HM and in teacher education. On the other hand, we understand the existence of a problematic, in which the HM books present a succinct, playful and scarce approach to the mathematical relations Fibonaccianas, little relevant for the reader to understand the evolutionary process of the MF from the description of the model reproduction of rabbits. In an epistemological perspective, this research has as investigation object the generating function and the characteristic equation, both are discussed in the class of the polynomial structures of the MF that compose a repertoire inherent to its complexification from the insertion of variables and the imaginary unit "i". In a didactic and cognitive approach, one intends to work these mathematical relationships in teaching situations. For that, the methodological course based on Didactic Engineering (ED), which is intrinsic to the researches in the teaching of Mathematics and which is carried out in didactic situations supported by the application of problem situations, is followed. Moreover, ED has as presuppositions epistemological, didactic and cognitive elements, thus, allowing to explore mathematical properties in HM classes. Finally, it was verified an internal validation of the research concerning the understanding of the generating function and characteristic equation. Moreover, it was possible to observe that some students, teachers in initial formation, developed an understanding of the historical and mathematical evolution of the Fibonacci Sequence.
\end{abstract}

Keywords: Didactic Engineering; History of Mathematics; Teacher training; Fibonacci complex model; Generating function.

\title{
Introdução
}

O experimento didático, discutido neste trabalho, foi realizado na disciplina de HM no curso de Licenciatura em Matemática do IFCE. Esse locus foi selecionado pelo interesse de inserir uma concepção epistemológica nas aulas de HM, tendo em vista que, segundo Alves e Borges (2011) e Alves (2015), os livros de HM discutem as relações Fibonaccianas, de maneira "lacônica, lúdica e desprovida de um vigor 
histórico-matemático que proporcione ao leitor um entendimento do processo evolutivo irrefreável hodierno do modelo de Fibonacci” (ALVES; CATARINO, 2016a, p.112).

Nos trabalhos de Alves (2015, 2016a, 2016b, 2016c), observa-se a descrição de um cenário, da HM, composto por "hiatos históricos" gerados a partir da escassa publicização das propriedades e teoremas oriundos da modelização da situaçãoproblema da produção de coelhos. À vista disso, esta pesquisa tem dois objetivos, o primeiro é de estudar a função geradora e equação característica, do modelo complexo de Fibonacci, em situações de ensino. E, o segundo é de instigar a compreensão de uma concepção epistemológica de conceitos matemáticos nas aulas de HM com a finalidade de contribuir na formação inicial de professores de Matemática.

Nesse sentido, questiona-se: qual delineamento metodológico possibilita realizar um experimento didático que oportunize explorar a função geradora e a equação característica, no contexto de investigação histórica do modelo complexo de Fibonacci? Desse modo, assumindo pressupostos epistemológicos, didáticos e cognitivos, define-se a ED como roteiro para a realização do experimento didático. A seguir, será discutida essa abordagem metodológica.

\section{Engenharia Didática}

A ED surgiu, no contexto da Didática da Matemática (DM) na França em 1980, como "um esquema experimental com base em realizações didáticas em sala de aula, isto é, na construção, realização, observação e análise de sessões de ensino" (ALMOULOUD, 2007, p. 171). Nesse sentido, o termo associa-se o percurso metodológico do professor-pesquisador como uma engenharia. Todavia, a ED possui pressupostos epistemológicos, diáticos e cognitivos (ARTIGUE, 1995, p. 98,).

Desse modo, Almouloud (2007, p. 149) explica que a epistemologia apresenta em seu escopo uma investigação, do processo de construção dos conhecimentos científicos, desde a sua origem histórica até seu atual estado de evolução. Na DM, conforme Pais (2002, p. 9), são investigados problemas que surgem na concepção de definições matemáticas e conceitos didáticos.

Além do mais, a análise epistemológica, no ensino de Matemática, recorre ao desenvolvimento de conceitos matemáticos, possibilitando categorizar as diversas concepções sobre um objeto de investigação. "Esse tipo de análise pode auxiliar o 
pesquisador em didática da matemática a entender melhor as relações entre os objetos matemáticos e controlar as variáveis didáticas relacionadas com o processo de ensino e aprendizagem de tais objetos" (ALMOULOUD, 2007, p. 156).

Pais (2002, p. 99) relata que a ED é um roteiro metodológico pelo qual o docente pode administrar a investigação e realizá-la em situações didáticas. Para isso, a ED é organizada em quatro fases consecutivas: análises preliminares, concepção e análise $a$ priori, experimentação, análise a posteriori e validação.

Conforme Pommer (2013, p. 23), nas análises preliminares, é feito um levantamento bibliográfico numa abordagem histórica dos elementos epistemológicos, didáticos e cognitivos do que se pretende ensinar em sala de aula e das concepções dos alunos. Destarte, a concepção e análise a priori designam o planejamento de situações de ensino amparadas na aplicação de situações-problema com o propósito de validar as hipóteses da pesquisa. Além disso, é feita uma predição do que se espera na fase de experimentação (ALMOULOUD, 2007, p. 174).

$\mathrm{Na}$ experimentação, as situações-problema são propostas aos alunos, diante disso, tem-se "um corpus de pesquisa variado, composto por produção dos alunos, registro de perguntas, dúvidas e erros constatados durante o acompanhamento de suas ações e diários de classe dos ministrantes" (CARNEIRO, 2005, p. 105). Por fim, a análise a posteriori e validação, tratam os dados coletados no sentido de comparar as situações descritas na análise a priori e a posteriori a fim de validar, internamente na situação de ensino, ou refutar as hipóteses de investigação (ALMOULOUD; SILVA, 2012, p. 27). A seguir, têm-se as análises preliminares.

\section{Análises preliminares inerentes ao modelo complexo de Fibonacci}

Nas análises preliminares, é feita uma revisão bibbliografica das relações matemáticas que compõem o repertório de representações complexas do MF. Desse conjunto, foram selecionadas, como objetos de investigação, a função geradora e a equação caracterítica. Com enfase na epistemologia matemática, conforme King (1963, p. 16), vale comentar que o MF foi criado para representar, matematicamente, a problematização da reprodução de coelhos. 
Dessa forma, o modelo original de Fibonacci é dado por $\mathrm{f}_{\mathrm{n}+2}=\mathrm{f}_{\mathrm{n}+1}+\mathrm{f}_{\mathrm{n}}, \forall \mathrm{n} \in \square$ para $\mathrm{f}_{0}=0$ e $\mathrm{f}_{1}=1$ (ALVES; CATARINO, 2016a). À vista disso, a evolução do modelo elaborado por Leonardo Pisano em 1202 permite investigar, nos compêndios da HM, outras representações Fibonaccianas, dentre as quais, as formas polinomiais se destacam. A priori, esses polinômios são definidos por Catalan e Jacobsthal em 1883.

No sentido de complexificar o MF, tem-se a definição $f_{n}(x)=x \cdot f_{n-1}(x)+f_{n-2}(x)$ $\operatorname{com}_{1}(x)=1, f_{2}(x)=x$ e $n \geq 1$ discutida por Brother (1963), Hoggatt e Long (1974) e Witford (1977). Doravante, encontra-se na literatura da Matemática Pura, de Hoggatt e Long (1974), Asci e Gurel (2012), Alves e Catarino (2016a, 2016b), uma extensão desses polinômios para representações bivariadas tal como: $F_{n+1}(x, y)=i x \cdot F_{n}(x, y)+F_{n-1}(x, y) \operatorname{com} F_{0}(x, y)=0, F_{1}(x, y)=1$ e $n \geq 1$.

No contexto das representações polinomiais, são discutidas as noções de função geradora $\mathrm{g}(\mathrm{t})=\sum_{\mathrm{n}=0}^{\infty} \mathrm{F}_{\mathrm{n}}(\mathrm{x}, \mathrm{y}) \cdot \mathrm{t}^{\mathrm{n}}$, equação característica $\mathrm{t}^{2}-\mathrm{ixt}-\mathrm{y}=0$ e definidas $t_{n+1}(x, y)=\frac{F_{n+1}(x, y)}{F_{n}(x, y)}$ e $\lim _{n \rightarrow \infty} \frac{F_{n+1}(x, y)}{F_{n}(x, y)}=t$ (ALVES; CATARINO, 2016a). A seguir, tem-se a concepção e as análises a priori referentes ao experimento didático.

\section{Concepção e análise a priori}

A priori, depois da investigação epistemológica na estrutura matemática de relações que compõem e determinam o modelo complexo de Fibonacci, é feita uma transposição, para o plano didático, de definições e propriedades a fím de realizar situações de ensino que permitam trabalhar a cognição do aluno, que neste caso é um professor de Matemática em formação, com o propósito de instigar o desenvolvimento de uma compreensão epistemológica do ensino de HM e um raciocínio inferencial quanto à construção de conceitos matemáticos Fibonaccianos. 
Quadro 1 - Situação-problema sobre a equação característica

Descreva uma equação característica para o modelo complexo de Fibonacci considerando as seguintes definições: $\quad F_{n+1}(x, y)=i x \cdot F_{n}(x, y)+F_{n-1}(x, y) \quad c o m$ $\mathrm{F}_{0}(\mathrm{x}, \mathrm{y})=0, \mathrm{~F}_{1}(\mathrm{x}, \mathrm{y})=1$ e $\mathrm{n} \geq 1$ e $\mathrm{g}(\mathrm{t})=\sum_{\mathrm{n}=0}^{\infty} \mathrm{F}_{\mathrm{n}}(\mathrm{x}, \mathrm{y}) \cdot \mathrm{t}^{\mathrm{n}}$. Em seguida, discuta qual aspecto histórico-evolutivo pode ser compreendido em relação ao modelo que prevê a reprodução de coelhos, formulada por Leonardo Pisano em 1202.

Fonte: Acervo da pesquisa

Nesse sentido, acontece a concepção de um experimento didático com a pretensão de possibilitar uma discussão inerente ao modelo complexo de Fibonacci com ênfase em duas definições: $F_{n+1}(x, y)=i x \cdot F_{n}(x, y)+F_{n-1}(x, y) \operatorname{com}^{2}(x, y)=0, F_{1}(x, y)=1$ e $n \geq 1$ e $\mathrm{g}(\mathrm{t})=\sum_{\mathrm{n}=0}^{\infty} \mathrm{F}_{\mathrm{n}}(\mathrm{x}, \mathrm{y}) \cdot \mathrm{t}^{\mathrm{n}}$ (ALVES; CATARINO, 2016a). Para isso, foi elaborada e aplicada a situação-problema (quadro 1).

Além do mais, nesta fase de análise a priori, é feita uma predição dos possíveis raciocínios que os estudantes venham a manifestar durante a experimentação. Assim, espera-se que os alunos compreendam as fases de construção da equação característica e identifiquem esse processo como um delineamento histórico-matemático que designa a evolução do MF a partir da inserção da unidade imaginária “i” e de variáveis. A seguir, a experimentação será discutida.

\section{Experimentação}

A aplicação desta pesquisa aconteceu na disciplina de HM no curso de Licenciatura em Matemática do IFCE. As soluções e discussões dos alunos são os dados que foram coletados por meio das gravações de áudios e registros de imagens. Neste trabalho, será discutida uma situação-problema (quadro 1). De início, o professorpesquisador apresentou à turma uma abordagem epistemológica da HM, no que diz respeito ao MF quanto a sua origem na problematização da produção de coelhos e sua representação matemática pela recursividade $\mathrm{f}_{\mathrm{n}+2}=\mathrm{f}_{\mathrm{n}+1}+\mathrm{f}_{\mathrm{n}}, \forall \mathrm{n} \in \square$ para $\mathrm{f}_{0}=0$ e $\mathrm{f}_{1}=1$ (ALVES; CATARINO, 2016a).

No segundo momento, numa perspectiva do processo de complexificação do MF, foi proposta a situação-problema (quadro 1). Com o intuito de resolvê-la, alguns 
alunos propuseram a inserção da unidade imaginária "i” e das variáveis no somatório, de modo que se tenha: (ixt).g(t)e $\left(y t^{2}\right) \cdot g(t)$. Essa formulação foi inspirada a partir da apreciação da estrutura de $F_{n+1}(x, y)=i x \cdot F_{n}(x, y)+F_{n-1}(x, y)$, na qual se ver a presença das variáveis x e y. Durante a investigação da série formal de potências se percebe a necessidade de inserir " $t$ ".

Destarte, a função geradora $g(t)=\frac{1}{1-i x t-y^{2}}$ foi determinada e teve que assumir $t_{n+1}(x, y)=\frac{F_{n+1}(x, y)}{F_{n}(x, y)}$ e $\lim _{n \rightarrow \infty} \frac{F_{n+1}(x, y)}{F_{n}(x, y)}=t$ para que se pudesse escrever a equação característica $\mathrm{t}^{2}-\mathrm{ixt}-\mathrm{y}=0$. Assim, encerra-se a discussão da situaçãoproblema avaliando, juntamente com os estudantes, o que essa construção representa para o MF. Na próxima seção, serão apresentadas as análises a posteriori e a validação desta pesquisa.

\section{Análises a posteriori e validação}

Os dados obtidos, na experimentação, são avaliados com a finalidade de validar a hipótese da pesquisa. Nesse sentido, observou-se que o estudo inerente à equação característica para o modelo complexo de Fibonacci caracterizou uma abordagem epistemológica da aula de HM. As discussões das relações matemáticas Fibonaccianas foram norteadas pela literatura de Alves e Catarino (2016a, 2016b), o que teve muita relevância na compreensão da construção e formalização da função geradora e equação característica.

Quadro 2 - Sistematização da série formal de potências

$$
\left\{\begin{array}{l}
g(t):=\sum_{n=0}^{\infty} F_{n} \cdot t^{n}=F_{0} \cdot t^{0}+F_{1} \cdot t^{1}+F_{2} \cdot t^{2}+\cdots+F_{n} \cdot t^{n}+\cdots \\
i x t \cdot g(t):=i x \cdot \sum_{n=0}^{\infty} F_{n} \cdot t^{n+1}=i x \cdot F_{0} t+i x \cdot F_{1} \cdot t^{2}+\cdots+i x \cdot F_{n-1} \cdot t^{n}+i x \cdot F_{n} \cdot t^{n+1}+\cdots \\
y t^{2} \cdot g(t):=y \cdot \sum_{n=0}^{\infty} F_{n} \cdot t^{n+2}=y \cdot F_{0} \cdot t^{2}+y \cdot F_{1} \cdot t^{3}+\cdots+y \cdot F_{n-1} \cdot t^{n+1}+y F_{n} \cdot t^{n+2}+\cdots
\end{array}\right.
$$

Fonte: Alves e Catarino (2016a, p. 115) 
Ainda na perspectiva epistemológica, foi explorado $g(t)-(i x t) \cdot g(t)-\left(y t^{2}\right) \cdot g(t)$ (quadro 2), tal que, recorrendo à $\mathrm{F}_{\mathrm{n}+1}(\mathrm{x}, \mathrm{y})=\mathrm{ix} \cdot \mathrm{F}_{\mathrm{n}}(\mathrm{x}, \mathrm{y})+\mathrm{F}_{\mathrm{n}-1}(\mathrm{x}, \mathrm{y})$, é possível simplificar os cálculos e obtém-se a função geradora $\mathrm{g}(\mathrm{t})=\frac{1}{1-\mathrm{ixt}-\mathrm{yt}^{2}}$. Além disso, assumindo $t_{n+1}(x, y)=\frac{F_{n+1}(x, y)}{F_{n}(x, y)}$ e $\lim _{n \rightarrow \infty} \frac{F_{n+1}(x, y)}{F_{n}(x, y)}=t$, tem-se a equação característica $t^{2}-i x t-y=0$.

À vista disso, o professor-pesquisador teve que intervir em determinadas etapas de formulação na resolução dos alunos (figura 1), todavia, essa situação didática permitiu trabalhar de forma implícita, a validade matemática da função geradora e da equação característica. Com isso, pode-se observar que os elementos de ordem didática e cognitiva não foram ignorados na realização desta pesquisa.

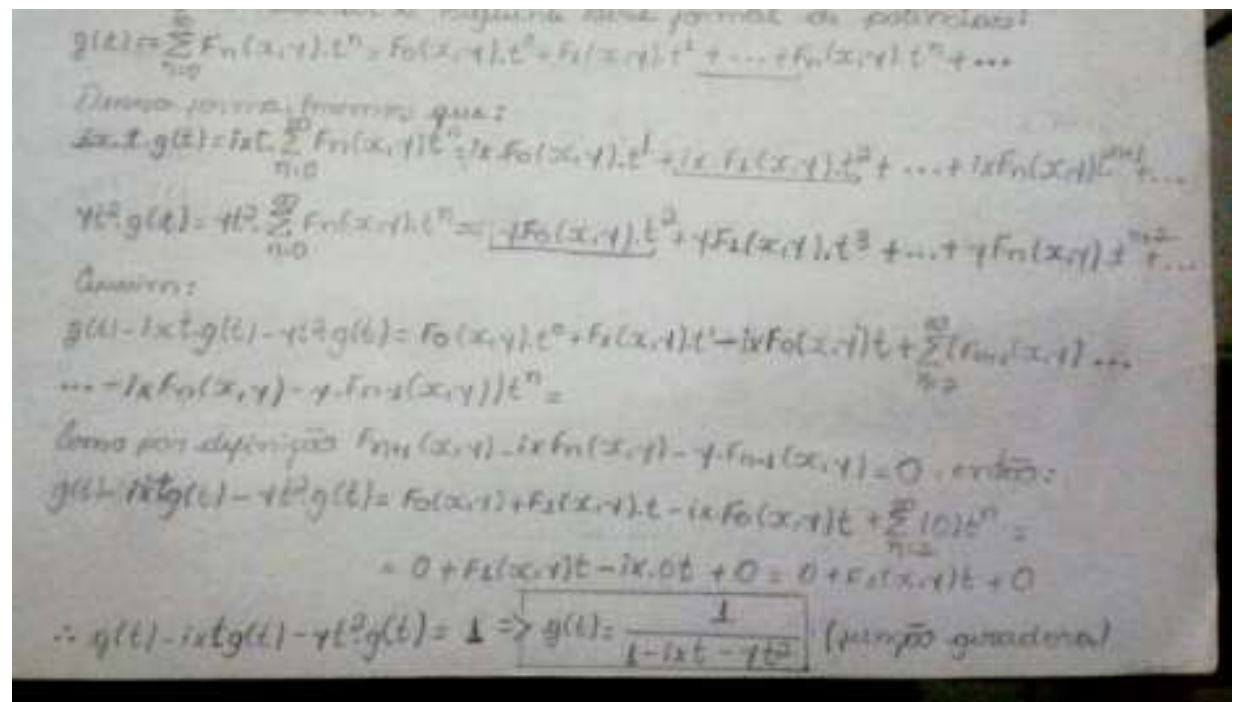

Figura 1 - Produção de um aluno na determinação da função geradora Fonte: Acervo da pesquisa

Além disso, alguns alunos, docentes em formação inicial, passaram a compreender que se pode trabalhar HM numa abordagem epistemológica e que o modelo complexo discutido, neste experimento didático, contribui para o processo histórico-evolutivo do MF. Portanto, alcançou-se uma validação interna desta pesquisa.

\section{Considerações finais}


O presente trabalho apresentou um experimento didático realizado em uma pesquisa de Mestrado do PGECM/IFCE e aplicado na disciplina de HM no curso de Licenciatura em Matemática do IFCE. Assim, apresentou-se uma discussão pormenorizada do processo de determinação da função geradora e da equação característica oriundas do modelo complexo de Fibonacci.

Essa situação é um fragmento da pesquisa que tem em seu escopo a investigação de representações complexas do MF. Dessa forma, é oferecido ao leitor um enredo metodológico que possui pressupostos epistemológicos, didáticos e cognitivos articulados, de modo que foi possível explorar as relações matemáticas no contexto de investigação histórica da complexificação do MF.

Além do mais, essa publicização feita na disciplina de HM, no contexto de formação docente, oportunizou o desenvolvimento de uma concepção epistemológica das aulas de HM. Doravante, espera-se que a compreensão da função geradora e da equação característica sirva de conhecimento prévio para o estudo de propriedades e teoremas pertencentes à família dos polinômios complexos e Fibonaccianos.

\section{Referências}

ALMOULOUD, S. A. Fundamentos da didática da matemática. Curitiba: UFPR, 2007.

ALMOULOUD, S. A.; SILVA, M. J. F. Engenharia didática: evolução e diversidade. Revemat: R. Eletr. de Edu. Matem., Florianópolis, v. 7, n. 2, p. 22-52, 2012.

ALVES, F.R.V. Sobre a evolução histórica do modelo de Fibonacci: a classe das funções hiperbólicas de Fibonacci. VYDIA Educação, v. 35, n. 1, p. 133-146, 2015.

ALVES, F.R.V. Sequência de Pell Generalizada - SGP: aspectos históricos e epistemológicos sobre a evolução de um modelo. Revista THEMA, v. 13, n. 1, p. 1-25, 2016 .

ALVES, F. R. V. Descobrindo definições matemáticas no contexto de investigação histórica: o caso da sequência generalizada de Fibonacci. BOLETIM GEPEM, n. 69, p. 1-7, 2016b.

ALVES, F. R. V. Engenharia Didática para a generalização da sequência de Fibonacci: uma experiência num curso de licenciatura. Educ. Matem. Pesq., São Paulo, v. 18, n. 1, p. 61-93, 2016c. 
Rannyelly Rodrigues de Oliveira, Maria Helena de Andrade e Francisco Régis Vieira Alves Função Geradora e Equação Característica no contexto de investigação histórica do modelo complexo de Fibonacci fundamentada na Engenharia Didática

ALVES, F.R.V; BORGES NETO, H. A existência de sequência de Fibonacci no campo dos inteiros: uma atividade de investigação apoiada nos pressupostos da Sequência Fedathi. BOLETIM GEPEM, n. 59, p. 135 - 140, 2011. Disponível em: <http://www.ufrrj.br/SEER/index.php?journal=gepem\&page=article\&op=view\&path\% 5B\%5D=647>. Acesso em: 06 nov. 2016.

ALVES, F. R. V.; CATARINO, P. M. M. C. A classe dos polinômios bivariados de Fibonacci (PBF): elementos recentes sobre a evolução de um modelo. Revista

THEMA, v. 14, n. 2, p. 112-136, 2016a.

ALVES, F. R. V.; CATARINO, P. M. M. C. THE BIVARIATE (COMPLEX) FIBONACCI AND LUCAS POLYNOMIALS: AN HISTORICAL INVESTIGATION WITH THE MAPLE'S HELP. Acta Didactica Napocensia, v. 9, n. 4, p. 71-95, 2016 b.

ARTIGUE, M., DOUADY, R., MORENO, L.; GÓMEZ, P. (Ed.). INGENIERÍA DIDÁCTICA EN EDUCACIÓN MATEMÁTICA. Un esquema para la investigación y la innovación en la enseñanza y el aprendizaje de las matemáticas. Bogotá: una empresa docente \& Grupo Editorial Iberoamérica, S.A. de C.V., 1995.

ASCI, M. \& GUREL, E. On bivariate complex Fibonacci and Lucas Polynomials. Notes on Number Theory and Discrete Mathematics, v. 18, n. 1, p. 1- 25, 2012.

BROTHER, U. A. Exploring Fibonacci Numbers. The Fibonacci Quarterly, v. 1, n. 1, February, p. $57-64,1963$.

CARNEIRO, V. C. G. Engenharia didática: um referencial para ação investigativa e para formação de professores de Matemática. Zetetiké, Campinas: UNICAMP, v. 13, n. 23, p. 85-118, 2005.

HOGGATT, V. E.; LONG, C. T.. Divisibility properties of generalized Fibonacci polynomials. The Fibonacci Quarterly. v. 12, n. 2, p. 113-121, 1974.

KING, C. Leonardo Fibonacci. The Fibonacci Quarterly. v. 1, n. 4, December, p. 1519, 1963.

PAIS, L. C. Didática da Matemática: uma análise da influência francesa. 2. ed. Belo Horizonte: Autêntica, 2002.

POMMER, W. M.. A Engenharia Didática em sala de aula: Elementos básicos e uma ilustração envolvendo as Equações Diofantinas Lineares. São Paulo, 2013. Disponível em:

<http://stoa.usp.br/wmpommer/files/3915/20692/Livro+Eng\%C2\%AA+Did\%C3\%A1ti ca+2013.pdf> Acesso em: 03 mar. 2017.

WITFORD, A. K. Binet's formula generalized. The Fibonacci Quarterly. v. 15, n. 1, February, p. 21-22, 1977. 